\title{
An oriented process induced by dynamically regulated energy barriers
}

\author{
Naoko Nakagawa \\ Department of Mathematical Sciences, Ibaraki University, Mito, Ibaraki 310-8512, Japan \\ Laboratoire de Physique, ENS-Lyon, 46 allée d'Italie, 69364 Lyon Cedex 07, France \\ Teruhisa S. Komatsu \\ Department of Physics, Gakushuin University, Mejiro, Toshima-ku, Tokyo 171-8588, Japan
}

(14 April, 2004)

\begin{abstract}
A novel mechanism for the appearance of oriented processes is investigated with a flexible dynamical system overcoming barriers. Under non-equilibrium condition with external driving, reaction paths deviate from that at equilibrium with an accompanying violation of symmetry between the forward and the reverse paths. Although we never introduce any external switching of potentials to generate the oriented processes, multidimensional flexible dynamics promote the oriented processes through this symmetry violation. Along the reaction paths, bottleneck points are proposed as a rate-controlling factor, which determine the direction-dependent activation energies satisfying Arrheniuslike law for the rate constants. In comparison, in stiff systems, the oriented process is suggested to appear in different manner from this scenario.
\end{abstract}

05.70.Ln 87.15.Aa 82.20.Pm

\section{INTRODUCTION}

In thermal equilibrium, free energy difference is considered as the single driving force to generate oriented processes. On the other hand, far from equilibrium, biomolecular processes are often said to be "efficiently" facilitated. Then other mechanisms to generate oriented processes may be explored in non-equilibrium situation. One possible example may be clearly found in a biomotor showing directional stepping motion along a filament [1]. The stepping of the motor is a kind of reaction shifting the binding site from one to the neighbors. Since its position does not contribute to the free energy, the directionality is not explained by the free energy difference between before and after this reaction. As ATP-hydrolysis drives the motor far from equilibrium, the stepping reaction may not be explained by the rules that govern equilibrium oriented reaction. Some experimental works [2,3] suggest that the effect of ATP-hydrolysis for the stepping reaction is to generate the direction-dependent activation energies. Such dependency certainly induces oriented process of the motor, but the question is then to understand how such a dependency can appear in non-equilibrium.

The generation of oriented processes is a problem of general importance in biological systems. Protein folding is facilitated far from equilibrium in some chaperonins with ATP-hydrolysis [4]. Although the folding processes are originally oriented by free energy difference, the possible facilitation mechanism would act additively to accelerate the processes. Quick conformational changes of proteins after excitations [5] might be facilitated in a similar way. Keeping several possible examples in mind, we want to propose mechanisms which lead to oriented processes. In this paper, we never introduce any external switching of potentials to generate the oriented processes, but, instead we show that multi-dimensional dynamics can promote an oriented process provided the system is far from equilibrium.

\section{MODEL}

Many chemical reactions can be modeled by barrier overcoming process. The shift of a particle in one dimensional periodic potential, a standard theoretical frame for particle current or diffusion [6], gives a model to consider steady state for barrier overcoming process. Brownian ratchet models [7] possess similar structure, and the stepping process of biomotor systems, too. While referring to these preceding studies, we introduce a new feature that the periodic structure is not fixed but fluctuates dynamically. 
Consider a rail on which stable binding sites of the particle are arranged periodically. When the rail is modeled as a flexible chain, the particle senses the interaction potential modulated by the temporal conformations of the rail, i.e. modulated by the dynamics of the system itself $[8,9]$.

Non-equilibrium situation is introduced by two heat baths, one of which with temperature $T_{1}$ is attached to the rail and the other with $T_{2}$ to the particle. Although this setup seems a little bit artificial, it has the clear advantage that a non-equilibrium steady state of the system is achieved. The resulting system may resemble to Feynmann's ratchet heat engine [10], but in the present model the internal degrees of freedom of the rail are explicitly considered.

A schematic picture of the model is shown in Fig.1. Independent variables are the position $x_{h}$ of the particle and those of the lattice sites $\left\{x_{i}\right\}$ for the 1D chain, where $i$ is the site index. The particle and each lattice site interact through the potential $V\left(x_{h}-x_{i}\right)$, which has asymmetric shape with one minimum as shown in Fig.1. The particle senses the summation of these potentials, $V\left(x_{h},\left\{x_{i}\right\}\right) \equiv \sum_{i} V\left(x_{h}-x_{i}\right)$. The time development is described by Langevin equations,

$$
\begin{aligned}
& \ddot{x}_{i}=-\gamma \dot{x}_{i}+\sqrt{2 \gamma k_{B} T_{1}} \xi_{i}(t)-K_{c}\left\{\left(x_{i}-i l\right)+\left(x_{i}-\frac{x_{i-1}+x_{i+1}}{2}\right)\right\}-\frac{\partial V\left(x_{h}-x_{i}\right)}{\partial x_{i}}, \\
& \ddot{x}_{h}=-\gamma \dot{x}_{h}+\sqrt{2 \gamma k_{B} T_{2}} \xi_{h}(t)-\frac{\partial V\left(x_{h},\left\{x_{i}\right\}\right)}{\partial x_{h}}
\end{aligned}
$$

driven by two heat baths $\left(T_{2} \geq T_{1}\right)$. We adopt sufficiently small values of the temperatures $T_{1}$ and $T_{2}$ comparing to the potential well depth of $V . \gamma$ is a friction coefficient and $\xi_{\alpha}(t)$ represents Gaussian white noise. We use the units as Boltzmann constant $k_{B}=1$. Although Eqs.(1) include inertia terms, qualitatively the same results are obtained in overdamped cases without these terms. $K_{c}$ is the spring constant for the chain with which each lattice site is connected to neighbors, and also to fixed ground, with the natural interval length $l$.

Hereafter two typical values of stiffness $K_{c}$ are used for the demonstration: at $K_{c}=0.5$, the flexibility of the chain greatly affects dynamics of the system (flexible system), while at $K_{c}=5.0$ the dynamics of the internal degrees of freedom for the chain are considerably suppressed similarly to the cases of certain Brownian ratchets (stiff system).

\section{RATE CONSTANTS DEFINED FROM TIME SEQUENCE}

A typical time sequence of the particle position is shown in Fig.2, in which we can observe each jump (stepping process) of the particle. As is seen there, the particle steps to both direction with equal probability when $T_{1}=T_{2}$, whereas oriented motion appears under the existence of temperature difference $\left(T_{2}>T_{1}\right)$. In the following, 'forward' direction is defined for each value of $K_{c}$ as the direction of the oriented motion in $T_{2}>T_{1}$, and 'backward' direction as the reverse direction. The forward and the backward stepping processes correspond to their reverse process each other.

Because most of the successive stepping events are sufficiently separated in time, each process could be regarded as a stochastic one. Then, it is natural to consider the probability of the steppings in a steady driven state. The long-term observation of the particle position gives an ensemble of data how long the particle has stayed at one lattice site after previous stepping event. Consequently we have a "population" of waiting states as a function of waiting time. Time evolution of such population could be analogous to chemical kinetics, and a rate constant for the occurrence of a step is introduced as follows.

Let $N_{s}(\tau)$ denote the number of events that the particle stays at a lattice site without stepping during a time longer than $\tau$ and $N_{F}(\tau)\left(N_{B}(\tau)\right)$ denote the number of events that the particle has stepped forward (backward) within $\tau$. The rate of the forward (backward) stepping $\kappa_{F}(\tau)\left(\kappa_{B}(\tau)\right)$ is defined as

$$
\frac{d N_{F}}{d \tau}=\kappa_{F}(\tau) N_{s}(\tau), \quad \frac{d N_{B}}{d \tau}=\kappa_{B}(\tau) N_{s}(\tau),
$$

where $d N_{F} / d \tau\left(d N_{B} / d \tau\right)$ is the number of the forward (backward) stepping events per unit time.

In the inset of Fig.2, $\kappa_{F}(\tau)$ and $\kappa_{B}(\tau)$ are shown for the case of $K_{c}=0.5$ for $T_{2}>T_{1}$. It is found that these rates are almost constant for sufficiently large $\tau$. For all cases examined with changing $T_{1}, T_{2}$ and 
$K_{c}$, converged values for $\kappa_{F}(\tau)$ and $\kappa_{B}(\tau)$ were obtained, which implies the process can be regarded as an independent stochastic process for long $\tau$. In the following, we let $\kappa_{F}$ and $\kappa_{B}$, which referred to the rate constants for "stepping reaction", denote the constant values of $\kappa_{F}(\tau)$ and $\kappa_{B}(\tau)$ for large $\tau$.

From the long time average of the stepping motion, the current and the diffusion coefficient of the particle are defined as $J \equiv \lim _{t \rightarrow \infty}\left\langle x_{h}(t)-x_{h}(0)\right\rangle / t, D \equiv \lim _{t \rightarrow \infty}\left\langle\left(x_{h}(t)-x_{h}(0)\right)^{2}\right\rangle / 2 t$. For all the cases examined numerically, $J$ and $D$ converged to finite values. In thermal equilibrium $\left(T_{1}=T_{2}\right)$, the particle simply diffuses along the chain without drifting $(J=0)$ all over the temperature range, while $J \neq 0$ in non-equilibrium $\left(T_{2}>T_{1}\right)$. Interestingly, the direction of the drift in the flexible system $\left(K_{c}=0.5\right)$ is opposite to that in the stiff system $\left(K_{c}=5.0\right)$ as shown in Fig.3. The convergence for $D$ means that the particle shows normal diffusion not only in equilibrium but also in non-equilibrium steady states.

If the particle steps stochastically, $J$ and $D$ should be related to the rate constants as follows.

$$
|J| \simeq l\left(\kappa_{F}-\kappa_{B}\right), \quad D \simeq \frac{l^{2}\left(\kappa_{F}+\kappa_{B}\right)}{2},
$$

where $l$ is a lattice interval of the chain. As is seen in Fig. 3, these relations hold well especially in low temperature. At high temperature, deviation from these relation occurs since the contribution from correlated successive stepping (multiple stepping) in small $\tau$ is not negligible. Thus, it is confirmed that the stepping process is well characterized by the rate constants $\left(\kappa_{F}, \kappa_{B}\right)$ at least within some lower temperature range.

\section{BOTTLENECK POINTS AS A RATE-CONTROLLING FACTOR}

For a simple chemical reaction, it is well known that rate constant $\kappa$ obeys Arrhenius law $\kappa \sim$ $\exp \left(-E_{a} / k_{B} T\right)$, where $E_{a}$ is called activation energy characterizing the reaction rate. In transition state theory (TST), the activation energy is purely determined from the information of the potential energy surface along the reaction coordinate without considering temperature effect. The minimum height of energy barrier, the difference of potential energy at the saddle point from that at the initial stable point along the reaction coordinate, corresponds to the activation energy [11].

Far from equilibrium, we need to define which quantity can be used as the temperature to characterize the process. Because the particle position $x_{h}$ is the important observable for the stepping process, the fluctuations of $\dot{x}_{h}$ in the staying state at a site would give the temperature. This is approximately equal to kinetic temperature of the particle, $T_{h} \equiv\left\langle\dot{x}_{h}^{2}\right\rangle$, because the stepping event is rather rare and $J$ is sufficiently small in the range of our analysis. Here $\langle\cdot\rangle$ means ensemble and/or long-time average. Under non-equilibrium condition $\left(T_{2}>T_{1}\right), T_{h}$ takes values such that $T_{2}>T_{h}>T_{1}$ due to the connection to the chain, while $T_{h}$ is equal to $T_{2}\left(=T_{1}\right)$ under equilibrium condition.

Let us consider the activation energy for the present system according to TST. From the fixed point analysis for Eq.(1) [12], the stable and the saddle points can be determined so that the potential difference $\Delta U_{0}$ between them is obtained straightforwardly. Note that $\Delta U_{0}$ is a constant independent of temperature, while depends on the flexibility of the system. In the flexible system, there is a cooperative effect between the particle and the rail to make the activation energy lower. In the thermal equilibrium cases in Fig.4, the obtained $\Delta U_{0}$ actually works well as the activation energy in the Arrhenius law, characterizing the stepping rates for the broad range of temperatures. However, once the system is put under the non-equilibrium condition, the rate constants deviate from the Arrhenius relation in equilibrium.

In the following, we try to get a modified version of the Arrhenius relation, applicable to non-equilibrium situation. In TST, the saddle point is the most important for the calculation of the activation energy. Although the usage of the saddle point itself might not be valid far from equilibrium, the idea that some bottleneck point in phase space is the rate-controlling factor should not be necessarily abandoned. How can we find such a "bottleneck point"? The paths for the stepping process is thermally fluctuating, but the ensemble of these paths would be recognized as tube-like clouds in a phase space. Then the most probable reaction path (MPRP) could be defined by averaging over the tube-like clouds. We suppose the bottleneck point would be defined as the saddle-like point along this MPRP.

In MPRP the particle position $x_{h}$, which is the major observable for the stepping process, would be the important coordinate in the phase space. According to this idea, we propose that the ensemble 
which determines the bottleneck point consists of the points in the paths satisfying the conditions for the potential derivatives by $x_{h}$ :

$$
\frac{\partial V\left(x_{h},\left\{x_{i}\right\}\right)}{\partial x_{h}}=0, \quad \frac{\partial^{2} V\left(x_{h},\left\{x_{i}\right\}\right)}{\partial x_{h}^{2}}<0,
$$

with the supplemental condition that the points have been passed almost synchronously with the actual stepping events. The condition (4) is more gentle than that for the saddle points because it neglects the derivatives in other directions $\left\{x_{i}\right\}$.

The first condition of Eqs. (4) means that force acting on the particle is zero at the point and the second condition means that the point is an unstable fixed point if looking only along $x_{h}$. By averaging the ensemble of the points satisfying the above condition with distinction of the direction of the stepping, bottleneck points for the forward and the backward stepping processes are obtained separately.

Interestingly, the bottleneck points for the forward and the backward stepping are often different in nonequilibrium $\left(T_{2}>T_{1}\right)$, while the two points is always identical in equilibrium $\left(T_{2}=T_{1}\right)$. It suggests that the symmetry between the forward and the backward processes is violated in the presence of temperature difference.

\section{DIRECTION-DEPENDENT ACTIVATION ENERGIES}

The difference of the bottleneck points will lead to the difference of the energy barrier. The barrier height $\Delta U_{F}\left(\Delta U_{B}\right)$ can be determined for the forward (backward) stepping process from the potential difference between the stable and the bottleneck point in similar manner to $\Delta U_{0}$. It is noted that $\Delta U_{F}$ and $\Delta U_{B}$ could depend on the temperatures in contrast to $\Delta U_{0}$, because the bottleneck point is obtained as the ensemble average of the points in the numerically simulated trajectories.

In Figs.5, $\Delta U_{F}$ and $\Delta U_{B}$ are displayed as a function of the external driving (temperature difference). In equilibrium $\left(T_{1}=T_{2}\right), \Delta U_{F}$ is equal to $\Delta U_{B}$ as directly noticed from the identity of the bottleneck points between the two directions. This is consistent with the equality of the rate constants $\kappa_{R}=\kappa_{L}$. In non-equilibrium, the barrier heights varies from its equilibrium values, and their dependences on the external driving are different between the flexible and stiff system as is seen in Figs. 5.

For the flexible system $\left(K_{c}=0.5\right)$, barrier heights grow with the increase of the external driving, where the deviation of $\Delta U_{F}$ from $\Delta U_{0}$ is smaller than that of $\Delta U_{B}$. As clearly seen in Fig. 6, substituting $\Delta U_{F}$ and $\Delta U_{B}$ for the activation energies, the rate constants in non-equilibrium can be reproduced by the Arrhenius-like form

$$
\kappa_{F}=A \exp \left(-\frac{\Delta U_{F}}{k_{B} T_{h}}\right), \quad \kappa_{B}=A \exp \left(-\frac{\Delta U_{B}}{k_{B} T_{h}}\right)
$$

where $A$ is a temperature-independent prefactor approximately common in the forward and the backward stepping. This relation indicates that the bottleneck points are major rate-controlling factors and that $\Delta U_{F}\left(\Delta U_{B}\right)$ is the substantial activation energy for the forward (backward) stepping process far from equilibrium. The knowledge of these activation energies is sufficient to characterize the oriented stepping process.

To be emphasized, the direction-dependent activation energies mean that absolutely different paths are selected between the forward and the backward stepping reactions. From the dependency of $\Delta U_{F}$ and $\Delta U_{B}$, the reaction path for the backward stepping process is implied to be more sensitive to the external driving than that for the forward. This sensitivity may be related to the property of the cooperative dynamics to realize the reaction path but this is open as a future problem.

For the stiff systems, the fluctuations of the rail is small and the cooperative dynamics between the particle and the rail are almost suppressed. As a result, the bottleneck points do not draw apart from the saddle point with increasing external driving, and the energy barriers defined by these bottleneck points only slightly increase compared to $\Delta U_{0}$ (see Fig.5(b)). Such slight variation cannot account for the deviation of the rate constants in non-equilibrium seen in Fig.4, so the barrier heights $\Delta U_{F}$ and $\Delta U_{B}$ does not explain the relative rate $\kappa_{F} / \kappa_{B}$. Further investigation for the stiff systems is not considered here, but it can be done in parallel to the Brownian ratchet models which has no flexibility of the chain $[13,7]$. 


\section{DISCUSSION}

In this Paper, we have discussed mechanisms to generate oriented process. Far from equilibrium in the flexible system, the direction-dependent activation energies (DAEs) account for the oriented process well. For the appearance of DAEs due to dynamical coupling between components, the symmetry violation between forward and its reverse reaction paths is a key feature, and it never occurs in equilibrium. Thus oriented processes due to DAEs cannot be explained from the equilibrium properties of the relevant systems. Instead, we should develop a direct understanding of non-equilibrium phenomena. The fact that Arrhenius-like form could be recovered in non-equilibrium steady state might suggest such new direction to find some robust structure to describe the system in non-equilibrium.

Direction-dependent activation energies are also obtained for the stepping process in Kinesin biomotors by analyzing the rate constants for the stepping motion in experimental sequences $[2,3]$. Comparing to the simple diffusion process without ATP-hydrolysis exhibited by Kinesin mutants [14] (proposed as an equilibrium system), the directionality in the normal functioning state seems to be generated by the suppression of the backward stepping process while leaving the rate of the forward process almost similar to that in equilibrium [3]. Interestingly, our flexible system also exhibits a similar tendency under external driving.

\section{Acknowledgment}

We are grateful to F.Oosawa, M.Nishiyama and Y.Taniguchi for stimulating discussion to motivate this work; M.Peyrard for fruitful discussion and critical reading of this manuscript; and K.Kaneko for perpetual discussion from the beginning of this project.

[1] R. D. Vale and R. A. Milligan, Science, 288, 88-95 (2000)

[2] M. Nishiyama, H. Higuchi and T. Yanagida, Nature Cell Biol., 4, 790-797 (2002)

[3] Y. Taniguchi, M. Nishiyama, Y. Ishii and T. Yanagida, Biophys J. 86, 410A (2004)

[4] W. A. Fenton and A. L. Horwich, Protein Science, 6 743-760 (1997)

[5] J. T. Sage, S. M. Durbin, W. Sturhahn, D. C. Wharton, P. M. Champion, P. Hession, J. Sutter and E. E. Alp, Phys.Rev.Lett., 86 4966-4969 (2001)

[6] H. Risken, in "The Fokker-Planck Equation", Springer-Verlag Berlin (1989)

[7] P. Reimann, Physics Reports, 361, 57-265 (2002)

[8] N. Nakagawa and K. Kaneko, Phys. Rev. E 67, 040901(R) (2003)

[9] N. Nakagawa and K. Kaneko, to appear in Physica A (2004)

[10] R. P. Feynmann, "Lectures in Physics, Vol.I", ed., by R. P. Feynmann, R. B. Leighton and M. Sands, Addison-Wesley Publishing Co. (1963)

[11] P. Hanggi, P. Talkner and M. Dorkovec, Rev. Mod. Phys., 62, 251-341 (1990)

[12] N. Nakagawa, K. Kaneko and T. S. Komatsu, Chaos 131032 (2003)

[13] K. Sekimoto, J.Phys.Soc.Jpn., 66, 1234-1237 (1997)

[14] Y. Okada and N. Hirokawa, PNAS 95 640-645 (2000)

[15] A. M. Berezhkovskii, V. Yu. Zitserman, D. Y. Yang, J. Kuo and S. H. Lin, Physica A 251, 399-429 (1998) 
FIG. 1. Profile of the model (refer to the text). Potential form is asymmetric in space, $V(\Delta x)=K_{h} \tanh (p(\Delta x-r)) / \cosh (d \Delta x)$, where interaction is confined mostly to the nearest lattice sites $\left(d^{-1}=0.25\right.$ with $\left.l=1\right)$. The degree of asymmetry is fixed at $p=10$ and $r=0.3$, while coupling strength $K_{h}=0.2$. In numerical simulation, the chain consists of 7 or 40 lattice sites with periodic boundary condition, while $\gamma$ is fixed at 0.1 .

FIG. 2. Typical time sequence of $x_{h}$ for $K_{c}=0.5, T_{1}=0.02, T_{2}=0.08\left(T_{h}=0.042\right)$ and $T_{1}=T_{2}=0.042$. (Inset): $\tau$ vs $\bullet \kappa_{F}(\tau), \circ \kappa_{B}(\tau)$ calculated according to the definition in Eq.(2), which show quick convergence.

FIG. 3. Current $J$ and diffusion coefficient $D$ for $T_{1}=0.02$ with various values of $T_{2}$. The guidelines are the relationship in Eq.(3). $J$ changes its direction from the flexible to the stiff system, where it is positive in $x_{h}$ for $K_{c}=0.5$ while negative for $K_{c}=5.0$.

FIG. 4. Arrhenius plots, $1 / T_{h}$ vs $\kappa_{F}, \kappa_{B}$. In non-equilibrium (neq), we fix $T_{1}$ at 0.02 while $T_{2}$ is widely changed, i.e., the points in (neq) equal to those in (eq) at $1 / T_{h}=50$. The dotted lines are the Arrhenius law $\exp \left(-\Delta U_{0} / T_{h}\right)$, where $\Delta U_{0} \simeq 0.15$ and 0.22 for $K_{c}=0.5$ and 5.0, respectively. Prefactor is a fitted constant. For $K_{c}=0.5$, $\kappa_{F}($ eq $)=\kappa_{B}($ eq $) \simeq \kappa_{F}\left(\right.$ neq) while $\kappa_{B}($ neq $)<\kappa_{B}($ eq $)$. For $K_{c}=5.0$, both $\kappa_{F}$ (neq) and $\kappa_{B}($ neq) are suppressed.

FIG. 5. The increase of the potential barrier $\Delta U_{F}$ and $\Delta U_{B}$. The barrier height grows with $T_{h}-T_{1}$ for the flexible system but it does not for the stiff system. Upper $(K c=0.5): T_{1}=0.02$ (circle), $T_{1}=0.01$ (rectangle). Lower $(K c=0.5): T_{1}=0.02$ (circle), $T_{1}=0.03$ (rectangle). At the equilibrium point $\left(T_{h}=T_{1}\right)$ for $K_{c}=0.5$, $\Delta U_{F}=\Delta U_{B}$ but there are slightly larger than $\Delta U_{0}$. This deviation might be due to 'saddle point avoidance' $[15]$.

FIG. 6. Exponential relationship between $\kappa_{F}\left(\kappa_{B}\right)$ and $\Delta U_{F} / T_{h}\left(\Delta U_{B} / T_{h}\right)$ for $K_{c}=0.5\left(k_{B}=1\right)$. Arrhenius-like relationship is recovered with regarding $\Delta U_{B}$ as the activation energy. 


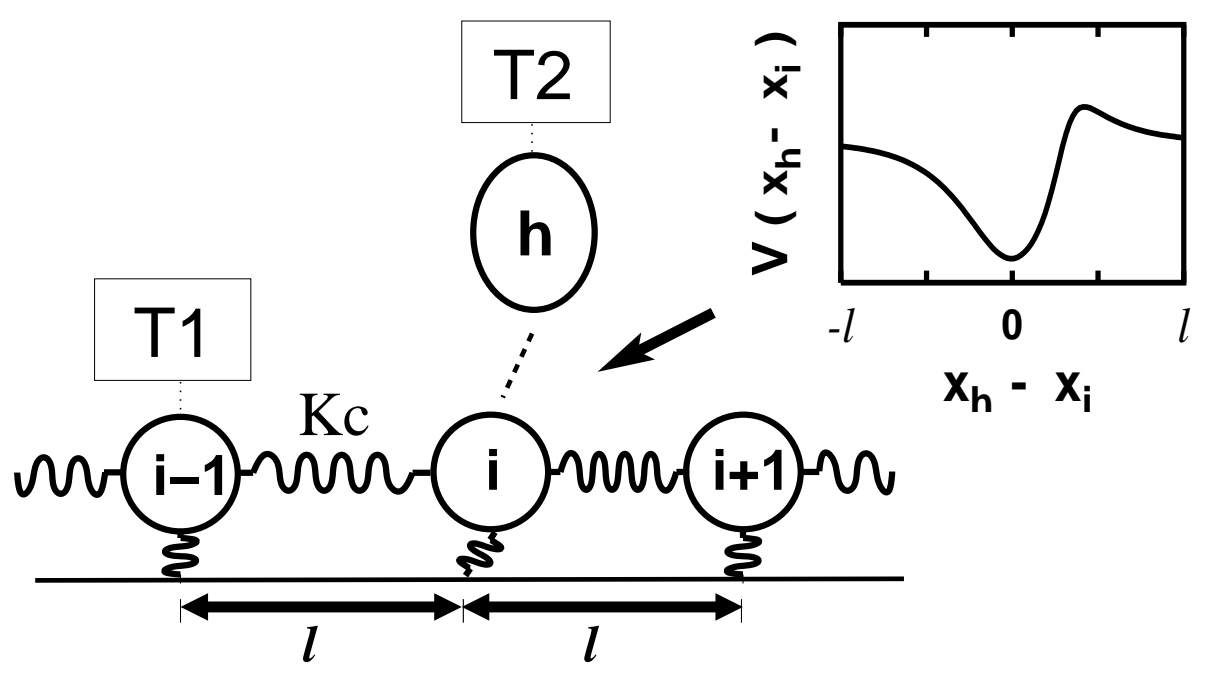

Nakagawa and Komatsu Fig.1 


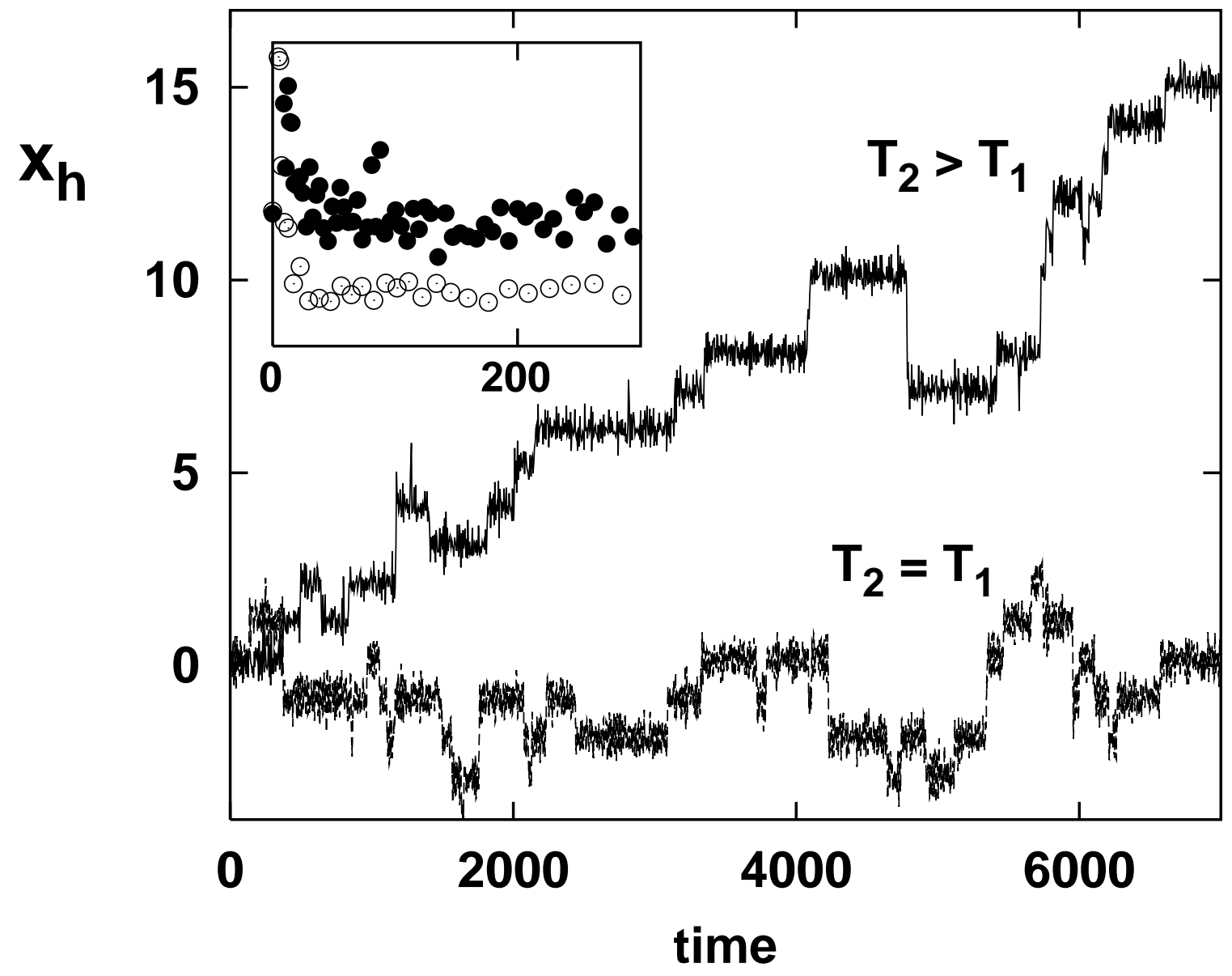

Nakagawga and Komatsu Fig.2 


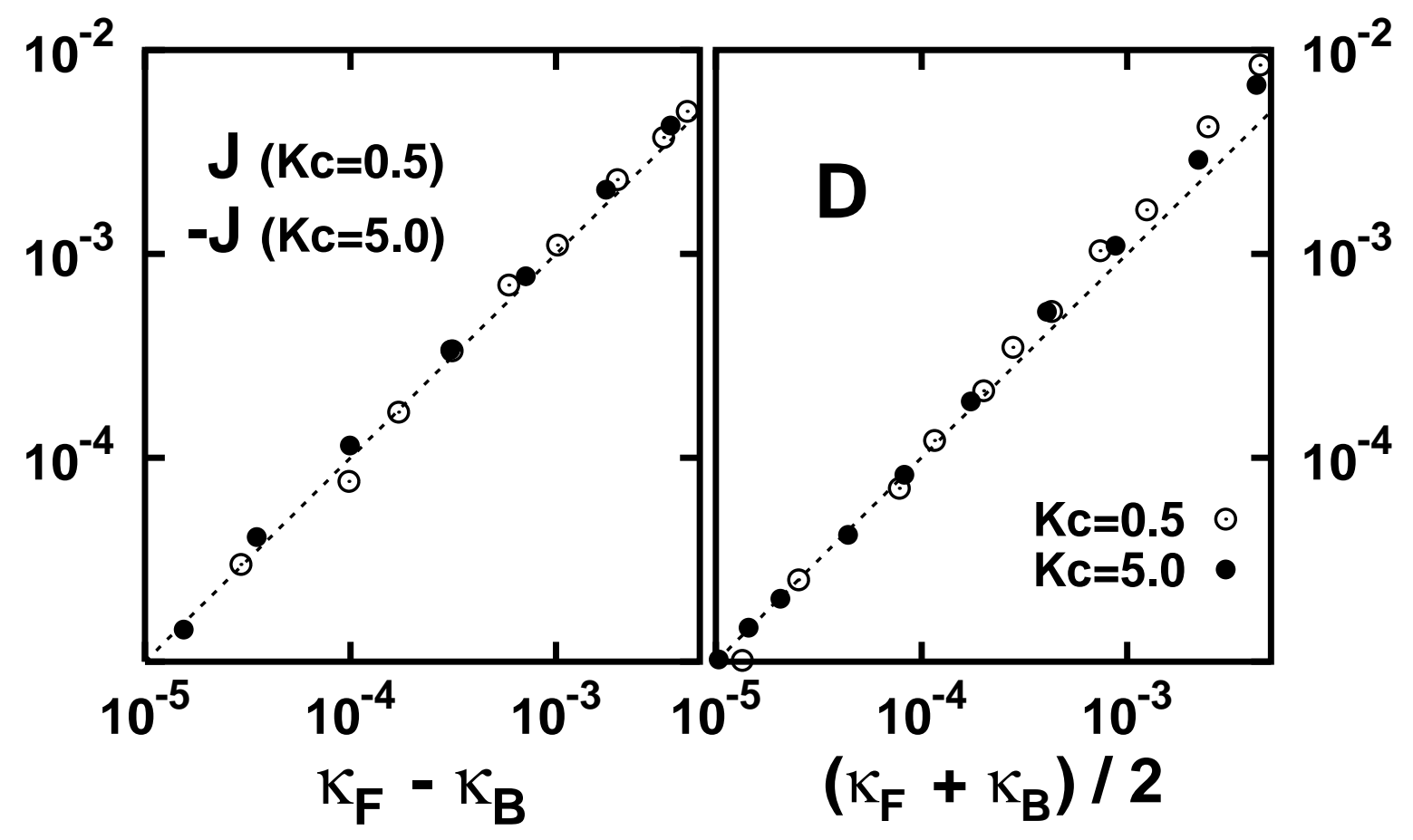

Nakagawa and Komatsu Fig.3 


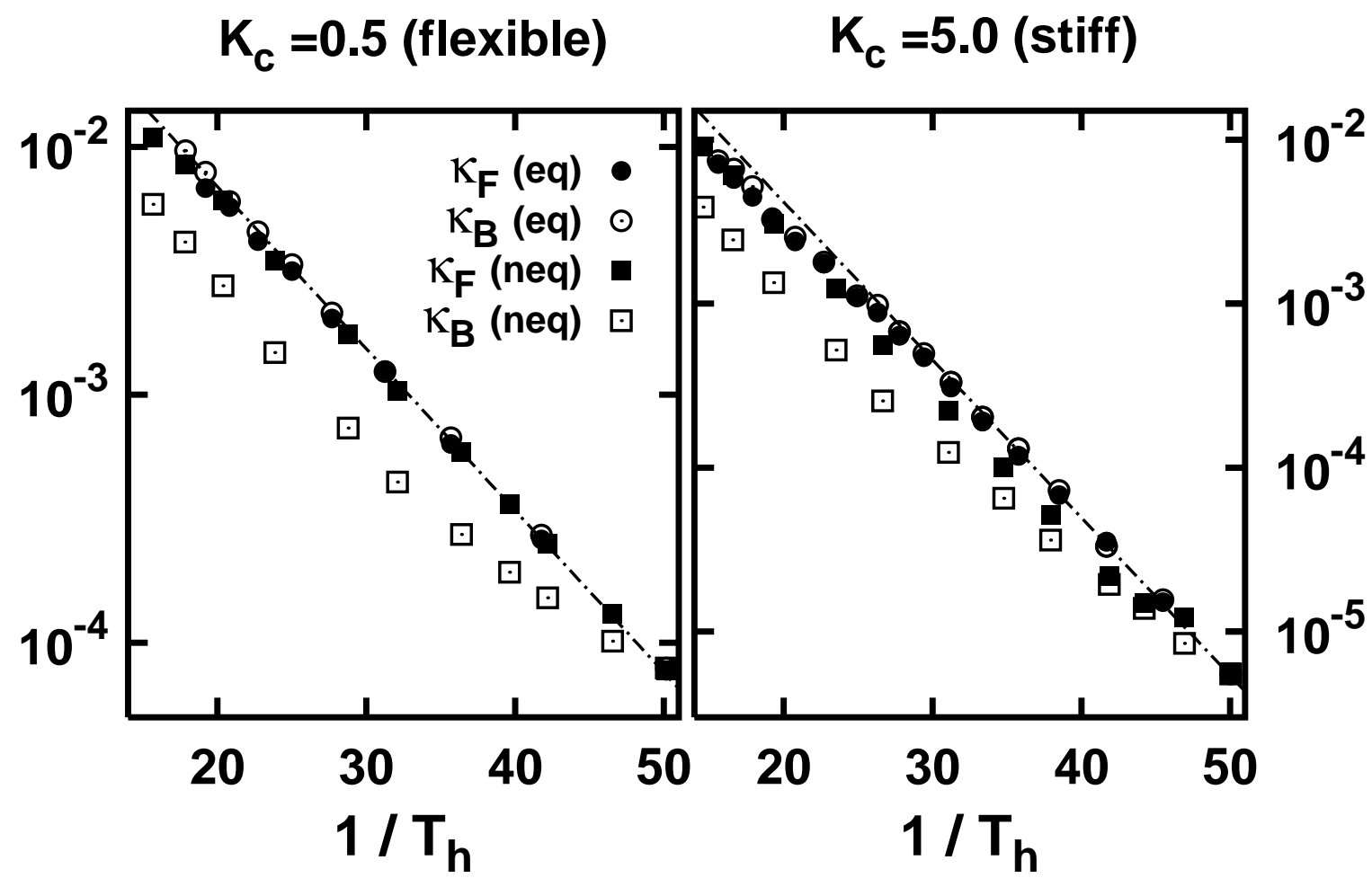

Nakagawa and Komatsu Fig.4 


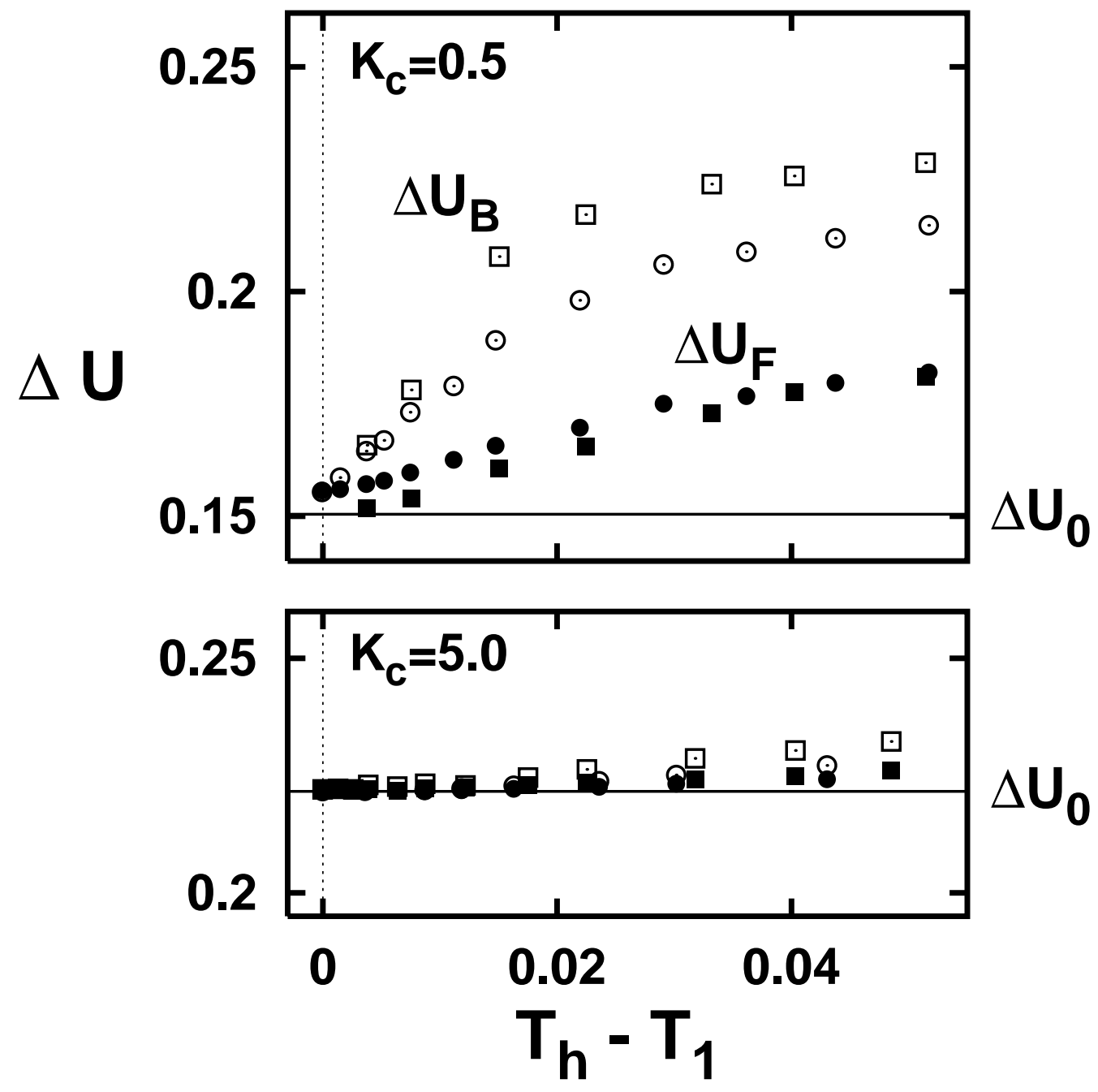

Nakagawa and Komatsu Fig.5 


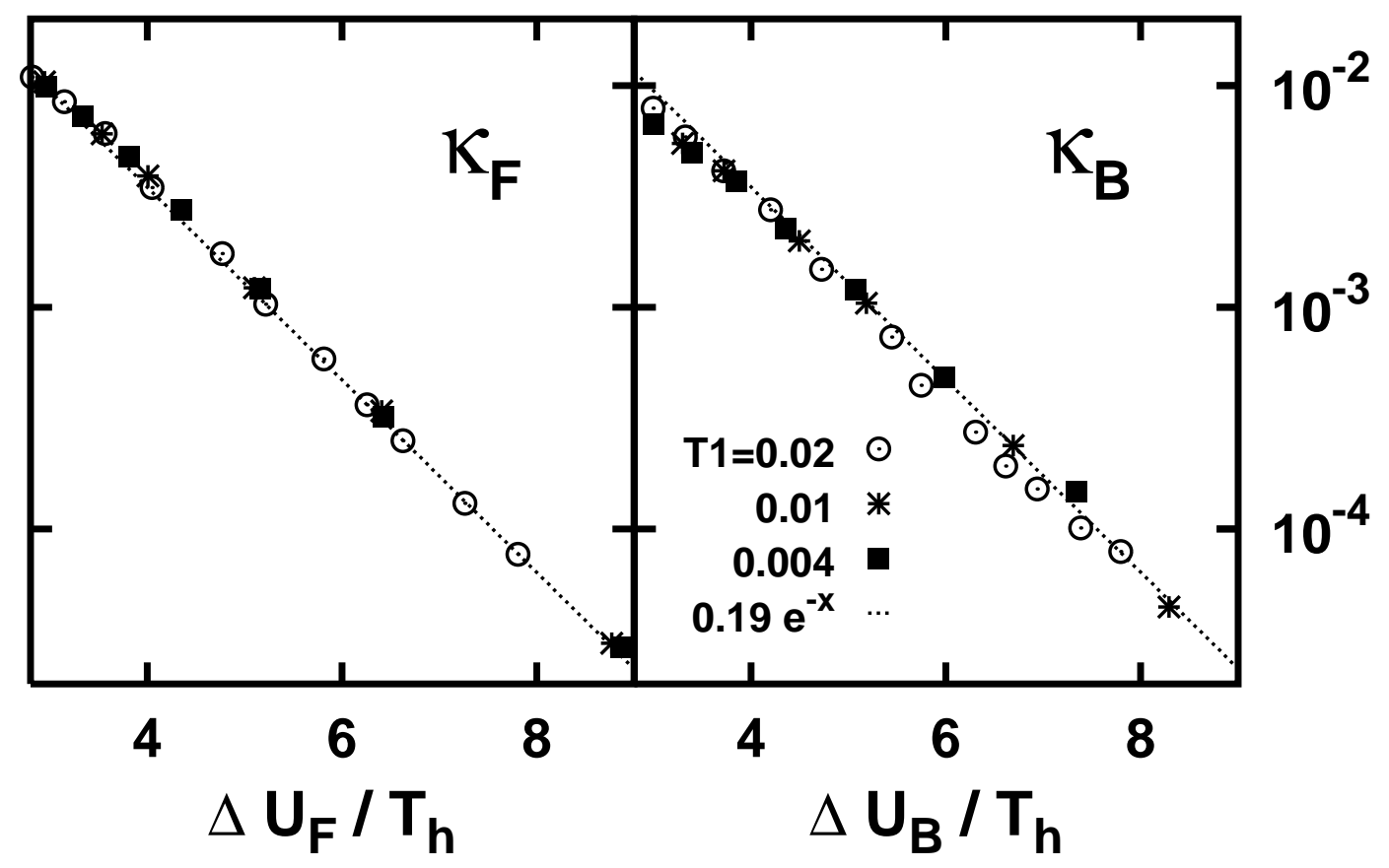

Nakagawa and Komatsu

Fig.6 